\title{
Floating-Wire Measurements of the Magnet Used for Large-Angle Pion-Nucleus Scattering at EPICS
}

\author{
W. B. Cottingame* \\ D. Oakley** \\ G. R. Burleson* \\ C. Milner** \\ M. Brown** \\ P. Seid!** \\ R. Kiziah** \\ N. Tanaka
}

\section{DISCLAIMER}

\begin{abstract}
This report was prepared as an account of work sponsored by an agency of the United States Government. Neither the United States Government nor any agency thereof, nor any of their employecs, makes any warranty, express or implied, or assumes any legal liability or responsibility for the accuracy, completeness, or usefulness of any information, apparatus, product, or process disclosed, or represents that its use would not infringe privately owned rights. Reference herein to any specific commercial product, process, or service by trade name, trademark, manufacturer, or otherwise does not necessarily constitute or imply its endorsement, recommendation, or favoring by the United States Government or any agency thereof. The views and opinions of authors expressed herein do not necessarily state or reflect those of the United States Government or any agency thereof.
\end{abstract}

-Guest Scientist at Los Alamos. New Mexico State University, Las Cruces, NM 88003.

** Guest Scientist at Los Alamos. University of Texas, Austin, TX 78712.

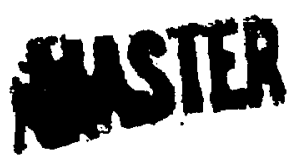


FLOATING-WIRE MEASUREMENTS UF THE MAGNET USED FOR

LARGE-ANGLE PION-NUCLEUS SCATTERING AT EPICS

by

W. B. Cottingame, G. R. Burleson, M. Brown, R. Kiziah, D. Dakley, C. Milner, P. Seidl, and N. Tanaka

\section{ABSTRACT}

Floating-jire measurements carried out on the circular magret used for a modification of the EPICS system for large-angle pion-nucleus scattering are described. The region of the incoming pion beam and that of the scattered pion were both covered. The effective radius of the magnet was determined to an uncertainty estimated to be $\pm 1.5 \mathrm{~mm}$. The resulting uncertainty in the scattering angle of the pion in experiments using this system is estimated to be $\pm 0.22^{\circ}$, with an upper limit of $\pm 0.50^{\circ}$.

\section{INTRODUCTION}

The purpose of this report is to describe the floating-wire measurements which were carried out on a magnet that was used in a modification of the EPICS system at LAMPF for studies of pion-nucleus scattering at large angles. The EPICS system ${ }^{l}$ consists of a plon channel that gives a large dispersed beam spot on a target, followed by a spectrometer used for analyzing the scattered pions which rotates around the target. The energy region covered by the system is $100-300 \mathrm{MeV}$. Before this work, the angular range of scattering covered by the spectrometer was limited to $\theta<120^{\circ}$. With this modification, the angular range which can be covered, for elastic scattering from a heavy nucleus, is $115^{\circ}<\theta<185^{\circ}$. The details of the method used will be described elsewhere.* A short description is given here.

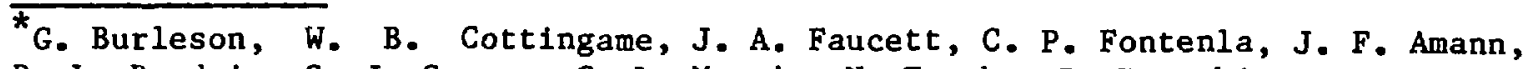
R. L. Boudrie, S. J. Greene, C. L. Morris, N. Tanaka, D. Yusnukis, M. Brown, R. Klziah, C. Milner, D. Oakley, C. Fred Moore, C. L. Blilie, S. J. SeestromMorris, D. Dehnhard, and K. Maeda, "Measurements of Large-Angle Pion-Nucleus Scattering," in preparation. 


\section{DESCRIPTION OF THE SYSTEM}

A top view of the magnet used for this modification 1s shown in Fig. 1 , and a side view is shown In Fig. 2. A magnet with 1-m diameter circular poles (named EURYDICE) from the LAMPF magnet pool was modified to allow for a 25-cm pole gap with a magnetic field in excess of $15 \mathrm{kG}$. This modification Involved the fabrication of new colls and of additional iron for the yoke. A new vacuum chamber, with provision for target storage and movement, was also fabricated. The magnet was located relative to the incoming beam and the spectrometer as indicated in Fig. 1. The pivot point of the spectrometer was moved downstream from its normal position by a distance of $52 \mathrm{~cm}$, and the spectrometer was moved further away from the target by $30 \mathrm{~cm}$, to allow space for the magnet.

The geometry of the central ray of the incoming beam as it passes through the circular magnet is shown in Fig. 3. The system is designed for this ray to pass through the center of the magnet, where the center of the target is located. To allow for this, the center of the magnet is offset a distance $D$ from the line of the incoming pion beam, as shown in the figure. The pion beam is bent through an angle $\psi$ both before and afte: passing through the center of the magnet, as shown in Fig. 3. If $R$ is the radius of effective fleld boundary of the magnet, the following relations apply:

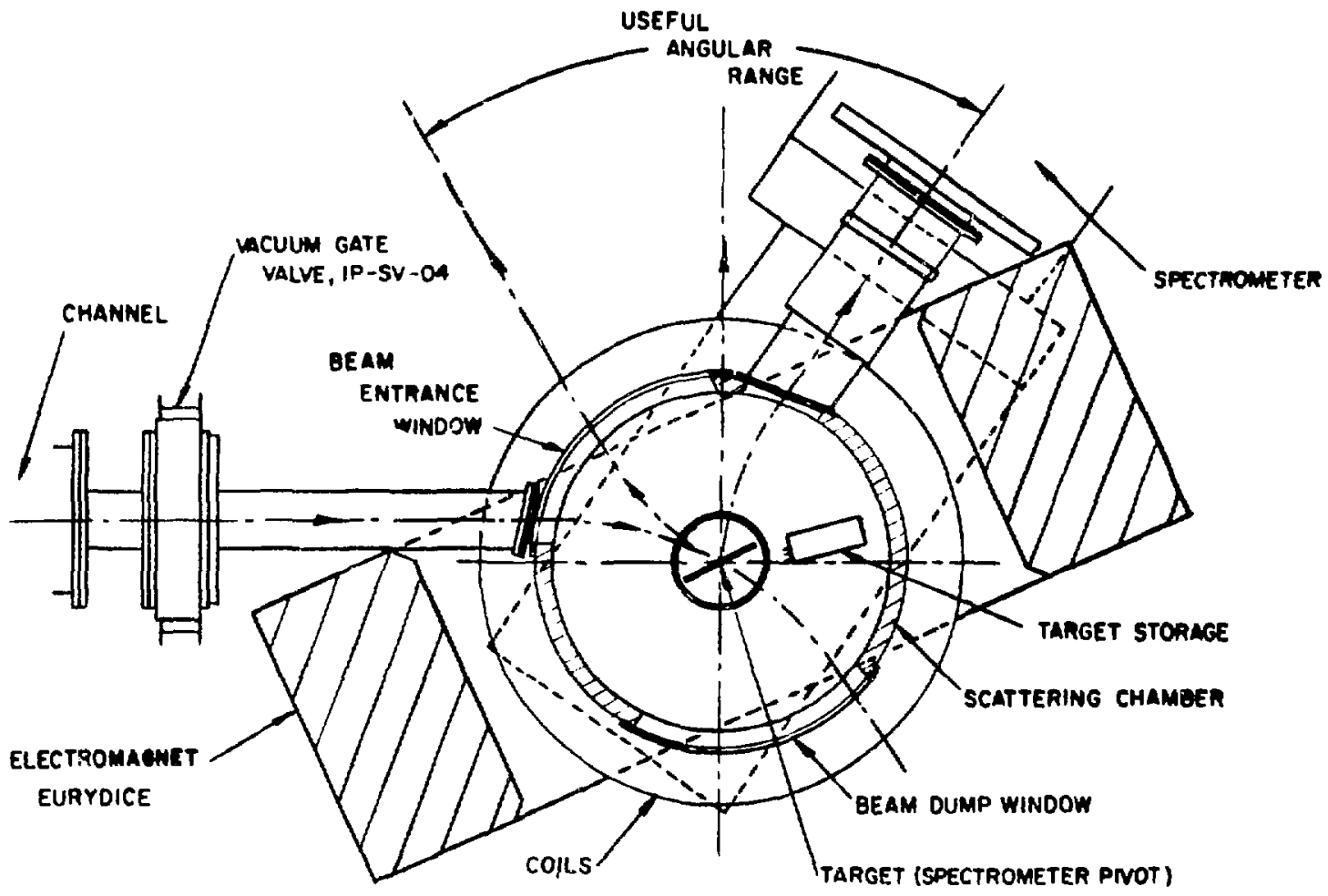

Fig. 1. Top view of the magnet system used for large-angle scattering at EPICS. 


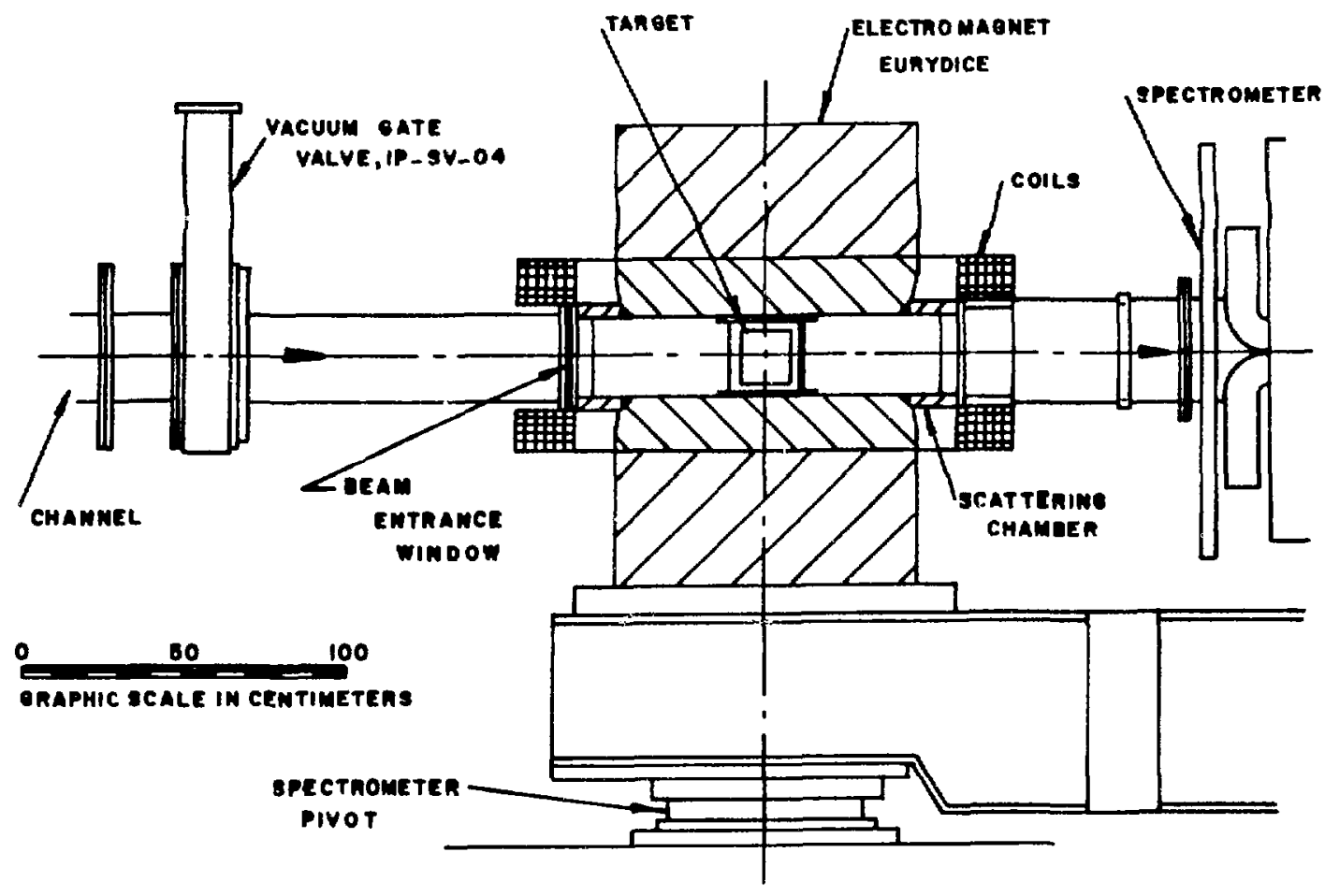

Fig. 2. Side view of the magnet system used for large-angle scattering at EPICS.

$D=R^{2} / 2 \rho$

and

$\sin (\psi / 2)=R / 2 \rho$

where the radius of curvature of the pion $\rho$ is related to its momentum $p$ by

$$
\rho=p / 0.3 B,
$$

with $\rho$ in $\mathrm{cm}, \mathrm{p}$ in $\mathrm{MeV} / \mathrm{c}$, and $\mathrm{B}$ the magnetic fleld in $\mathrm{kG}$. The design values of these quantities were $R \sim 53.3 \mathrm{~cm}, \rho \sim 103 \mathrm{~cm}, \psi \sim 30^{\circ}$, and $\mathrm{D} \sim 13.8 \mathrm{~cm}$, with $B \sim 13.5 \mathrm{~kg}$ for $300-\mathrm{MeV}$ plons.

The geometry for both the Incident and scattered pion is shown in Fig. 4. The geometry for the scattered pion is essentially the same as that for the incoming pion but :otated by an angle $\psi_{s^{*}}$. The symbols $R^{-}, D^{-}, \rho^{-}$, and $\psi^{-}$ designate the values of the analogous quantities, discussed above, for the incoming pion. (With azimuthal symmetry in the circular magnet, $\mathrm{R}^{-}$should be the same as $R_{.}$) The scattering angle of the pion $\theta_{s}$ is given by 


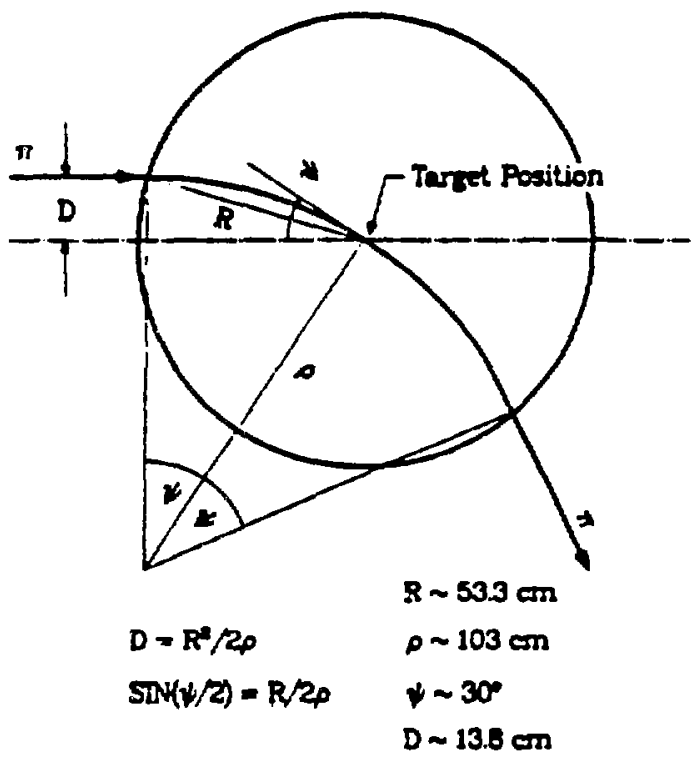

Fig. 3. Geometry of the central ray of the incoming beam passing through the circular magnet. The symbols used are described in the text.

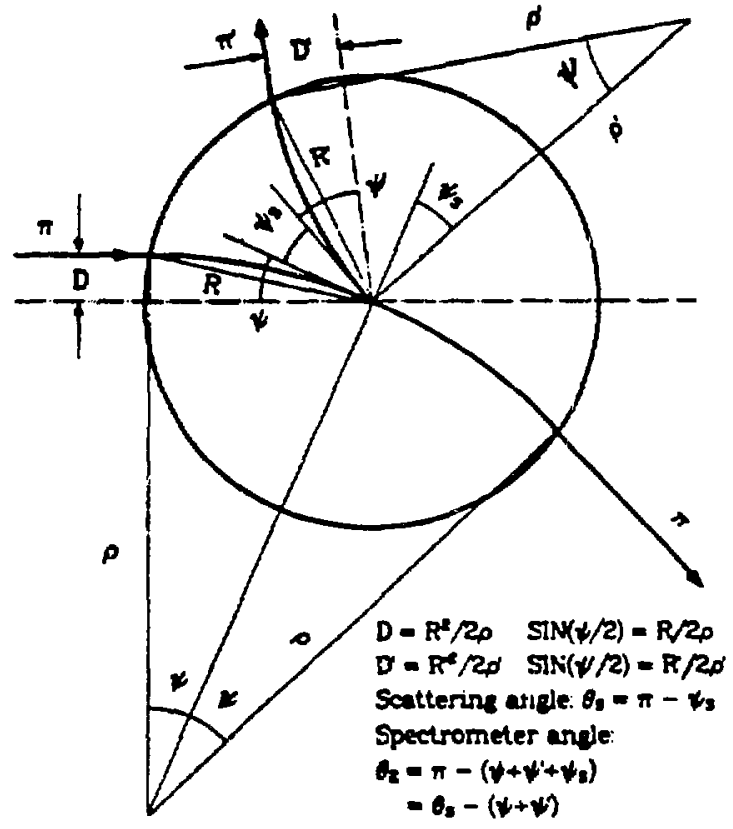

Fig. 4. Geometry of the central ray of the incoming beam, together with that of a pion scattered through an angle $\theta_{s}$. The symbols used are described in the text and in the figure.

$$
\theta_{s}=\pi-\psi_{s},
$$

and the angle of the EPICS spectrometer $\theta_{E}$ is

$$
\theta_{E}=\pi-\left(\psi+\psi^{-}+\psi_{S}\right)=\theta_{s}-\left(\psi+\psi^{-}\right) \text {. }
$$


The circular magnet is fixed with respect to the the EPICS spectrometer and rotates with it. The values of $R^{-}, D^{-}, \rho^{-}$, and $\psi^{-}$are set by the hardware design of the system, which ties the path of pions scattered from the center of the target to the central ray of the spectrometer. This means that in the experiment the field of the circular magnet must be set according to the momentum of the scattered pion, and the value of the offset $D$ of the incoming beam must be adjusted so that the central ray passes through the center of the target. Thus the scatcered pions detected in the spectrometer all pass through the same region of the magnetic field of the circular magnet, so that the effective solid angle of the spectrometer system $1 \mathrm{~s}$ independent of the scattering angle, an important feature of its design.

For proper operation of the system, it is necessary to know with some precision the magnetic properties of the circular magnet and, in particular, the value of the effective radii $R^{-}$for the outgoing pion and $R$ for the full region of the incoming beam, over the full energy range of the operation of the channel. This information determines the scattering angle $\theta_{s}$ observed in the experiment, since there is no simple method of finding this angle independently with the spectrometer system. The values of $R$ and $R^{\prime}$ were determined by the floating-wire measurements described below.

\section{THE FLOATING-WIRE MEASUREMENTS}

The floating-wire method is commonly used to determine the effects of magnets on moving charged particles, and it is described in several books. ${ }^{2}$ If a thin, flexible, wire of negligible mass carrying a current 1 is suspended under a tension $T$ in a magnetic field $B$, it will obey the same differential equation as that of a particle with charge $q$ and momentum $p$ passing through the field. This means that the path taken by such a particle passing through the maguet, or a portion of it, will be idertical to that of the shape assumed by the wire. The relation between these quistities can be $s$ hown ${ }^{2}$ to be

$$
\mathrm{p} / \mathrm{q}=\mathrm{T} / \mathrm{i}
$$

For $q$ equal to the the charge on the electron, $T$ in grams, $i$ in amperes, and $p$ in $\mathrm{MeV} / \mathrm{c}$, the relation is

$$
p=2.9388 \mathrm{~T} / 1
$$

In these measurements, the quantities to be determined were the effective radii $R$ and $R^{-}$. The method used was to measure a displacement $D$ (or $D^{-}$) from a parallel line passing through the center of the magnet of a wire with tension $T$ and current 1 extending from the geometrical center of the magnet to a region of negligible magnetic field. The effective momentum $p$ was determined from Eq. (7), the effective radius of curvature $\rho$ from $\mathrm{Eq} .(3)$, and the effective radius $R$ (or $R^{-}$) was determined from $E q$. (1). All measurements 
were carried out with the magnet in place in the EPICS system. All measurements of the magnetic fleld $B$ were made with a LAMPF-designed nuclear magnetic resonance (NMR) probe permanently located in a holder near the center of the magnet that was initially used for measuring a magnetization curve of the circular magnet. This same probe was used to set the fleld of the magnet during the experiment.

The wire used was made of beryllium copper and had a diameter of $0.10 \mathrm{~mm}$ (4 mils). It was anchored in the center of the magnet with a fixture that allowed it to rotate through an angle of about $\pm 30^{\circ}$ without causing $a$ bend or a kink in the wire. At the other end, it passed over a light-weight aluminum pulley which rotated with negligible friction. The fixture was mounted on a pivot to allow rotation around a vertical axis with negligible friction. The tension was provided by calibrated weights. Values between 50 and $95 \mathrm{~g}$ were used; the larger one corresponded nearly to the maximum tension that could be sustained by the wire. The current $i$ (typically 0.5 to $0.9 \mathrm{~A}$ ) was measured by noting the potential difference across a calibrated shunt with a digital voltmeter.

To measure the trajectory of the scattered pion, the wire was passed through the first three quadrupoles on the EPICS spectrometer. The magnetic axes of the first and third quadrupole were located by placing a transparent cell containing a colloidal suspension of ferrous oxide powder on the axis of the quadrupole, turning on the magnetic fleld, and sighting the center of the pattern produced in the cell by the quadrupole fleld of the magnet with a transit. This line was later transferred to transparent plexiglas fixtures which were located prinr to the first quadrupole and after the third quadrupole and were used to verify alignment on the axis. The quadrupoles were turned on at the end of the measurement to insure that there was no additional motion of the wire. This procedure avoided any problems related to a possible error in location of the wire with respect to the axis of the spectrometer.

For measurements corresponding to the incoming beam, a cart containing the pulley mounted on a screw adjustment taken from a lathe bed was placed a distance of about $5 \mathrm{~m}$ from the center of the magnet. The screw allowed for precise lateral motion of the end of the wire. The displacement $D$ from a parallel wire passing through the center of the magnet was read by observing the wire as 1 t passed over two machinist's rulers spaced $105 \mathrm{~cm}$ apart along the wire. Only a single wire was used, whose position was noted on the two rulers with and without current passing through the wire. The use of the two rulers assured that the wire was displaced parallel to its original line when the current was passed through the wire. We estimate that the position of the wire could be read to $0.5 \mathrm{~mm}$ with this method. For the measurements, the value of the current passing through the wire was varied, for a constant magnetic fleld, until the value of the offset $D$ was $13.85 \mathrm{~cm}$. To vary the angle, the magnet was rotated by rotating the spectrometer mount. The range of angle that: could be covered corresponded to a scattering angle of $95^{\circ}<\theta_{s}<150^{\circ}$, since the layout of equipment in the EPICS spectrometer area prohibited measurements extending to larger angles at that time. To find the effect of the pion beam passing near the iron yoke of the magnet for scattering at the largest angles, the current in the wire was reversed in order to push it to a region near the yoke. For this measurement, the wire was about $15 \mathrm{~cm}$ from the yoke, closer than the central ray of the beam for $180^{\circ}$ scattering, which is $28 \mathrm{~cm}$. The value of the effective radius $R$ measured here was consistent with the other values found. 
The vertical position of the wire was at beam level for most cf the measurements. Some misalignment of the system was noted, however. In a survey with transits, the spectrometer quadrupoles were found to $\mathrm{h} s \sim 1.6 \mathrm{~cm}$ below beam level at its front alignment mark and $\sim 1.4 \mathrm{~cm}$ below beam level at its rear alignment mark. (These values were a function of the angular position of the spectrometer.) The central plane of the circular magnet was also measured to be $0.325 \mathrm{~cm}$ above the bean height.

\section{RESULTS}

The magnetization curve of the circular magnet is shown in Fig. 5, which is a plot of the field $B$ as a funcicion of the voltage reading on a shunt on the magnet power supply and of the current in the magnet. Readings were taken for both increasing and decreasing currint to show the hysteresis effect, which is small, giving an average difference of $\sim 45$ gauss for flelds between 9 and $15 \mathrm{kG}$. Saturation effects are seen for fields above $\sim 12 \mathrm{kG}$, corresponding to pion energies above $\sim 250$ Miel.

For measurements of the wire running through the quadrupoles, the results for the value of the effective radius $R^{\prime}$ of the scattered pion as a function of pion momentum (or magnetic field) for three values of the tension $T$ in the wire are shown in Fig. 6. These values assume that the offset $D^{-}$is equal to its design value of $13.8 \mathrm{~cm}$. This curve indicates that the value of $R^{-}$is about $55.2 \mathrm{~cm}$ around $300 \mathrm{MeV} / \mathrm{c}$ ( $200 \mathrm{MeV}$ incident pion energy), possibly increasing for smaller momenta and decreasing somewhat for momenta above 360 $\mathrm{MeV} / \mathrm{c}$ (Incident pion energy $\sim 250 \mathrm{MeV}$ ), where saturation effects in the magnet begin.

The results for the effective radius $R$ as a function of scattering angle (for an infinitely heavy target nucleus) for five values of pion momentum between 225 and $410 \mathrm{MeV} / \mathrm{c}$ are shown in Fig. 7. Within the experimental uncertainly, there is no clear evidence for any azimuthal asymmetry, but there is an indication of a small decrease in $R(\sim 0.25 \mathrm{~cm})$ with increasing momentum. The value found for $R$ is about $57.05 \mathrm{~cm}$, larger than the $55.2 \mathrm{~cm}$ value that was found for $R^{-}$. The reason for this difference is not understood at this time. To check the effect of the misalignment of the circular magnet with respect to the beam helght, some neasurements of $R$ were repeated with the vertical position of the wire lowered to the height of the intersection of the line thiough the center of the spectrometer with the beam height; no measurable difference in the value of $R$ was found.

The uncertainty in the experimental pion scattering angle $\theta_{\mathrm{s}}$ can be estimated from the uncertainties in the various quantities measured in this work using Eqs. (1) - (6). We take the following estimates: magnetic field, $B, 0.1$ percent; current in the wire, $i, 0.02$ percent; tension in the wire, $T$, 0.2 percent, and the of $f$ set, $D, 0.7 \mathrm{~mm}$. This gives an overall uncertainty in the effective radius $R$ (or $R^{-}$) of $\pm 1.5 \mathrm{~mm}$, and in the scattering angle $\theta_{s}$ of $\pm 0.22^{\circ}$. Another estimate of the error on the scattering angle can be found by taking the uncertainty in $R$ to be equal to the full $6-\mathrm{mm}$ spread in its values found during the measurements; this gives an uncertainty in $\theta_{s}$ of $\pm 0.16^{\circ}$. An upper limit can be estimated by assuming that the $2-\mathrm{cm}$ difference found between $R$ and $K^{-}$is incorrect, resulting from systematic errors; this would correspond to an error in $\theta_{s}$ of $\pm 0.50^{\circ}$. 


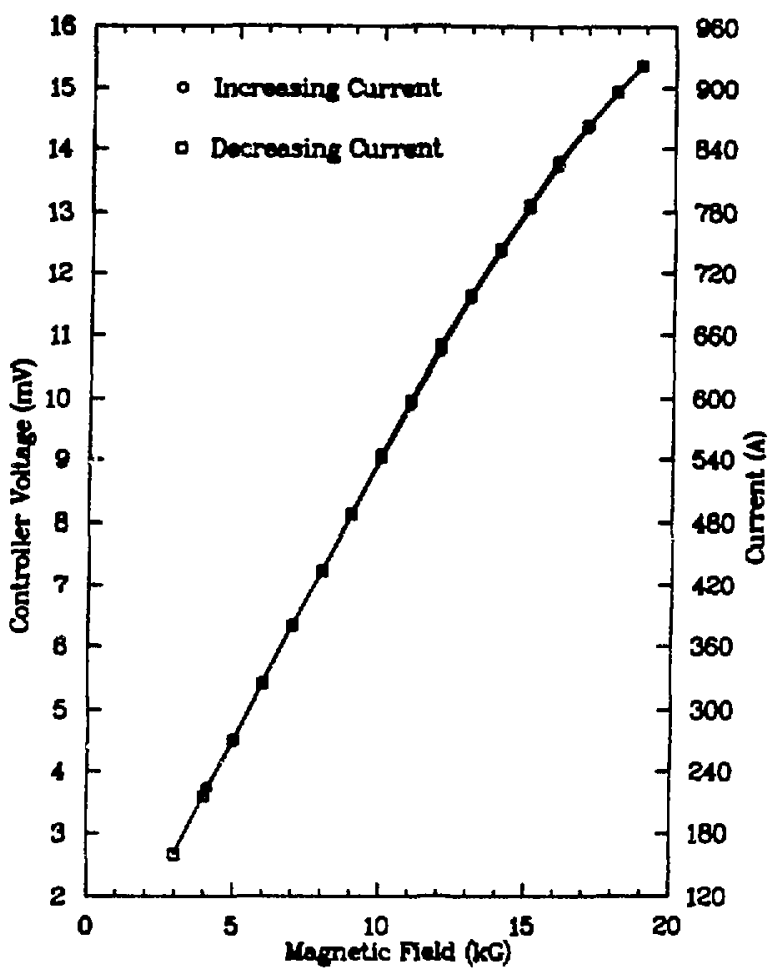

F1g. 5. The measured magnetization curve of the circular magnet, for both increasing and decreasing current. The magnet field $B$ is plotted as a function of the voltage on the shunt on the magnet power supply, as well as of the current in the magnet.

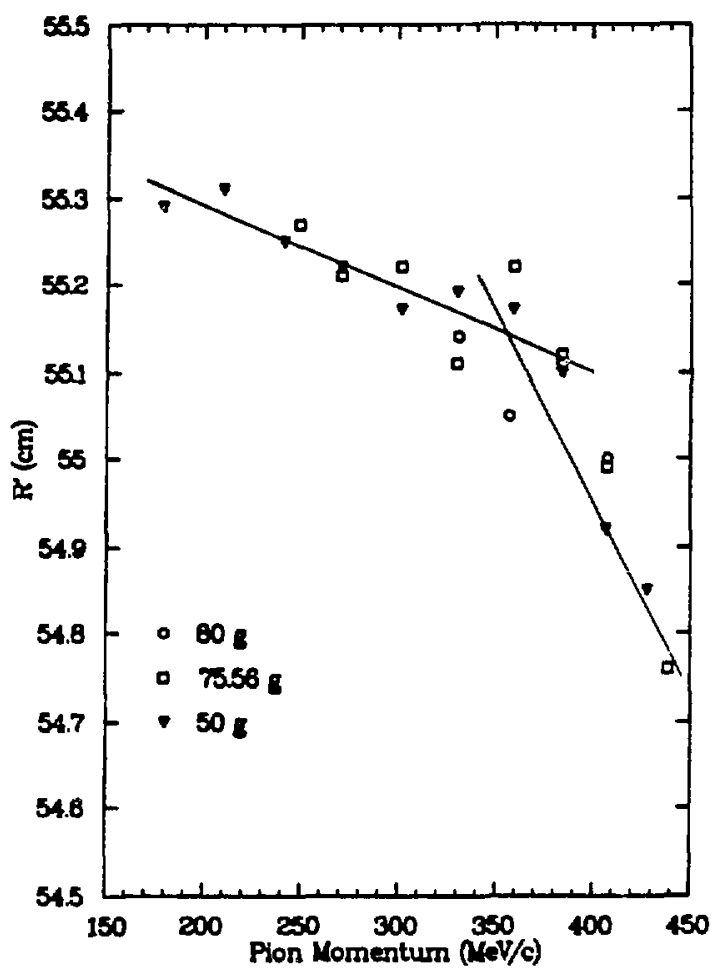

Fig. 6. Measured values of the effective radius $R^{\prime}$ of the circular magnet for the scattered pion as a function of pion momentum. Results are shown for three values of the tension $T$ in the wire, as indicated in the figure.

\section{REMARKS}

While the results of this work are generally, satisfactory and tend to confirm the important design features of the system, such as little azimuthal asymmetry of the magnet over the region of the incoming beam, there are features that we would like to investigate further. These include studies over a larger angular range and a confirmation of the difference between $R$ and $R^{-}$, and perhaps an understanding of the reason for 1 . In order to do this, we currently plan to carry out more wire-orbit measurements of the system during fall 1984, both with the magnet in place in the EPICS system and with the magnet alone. 


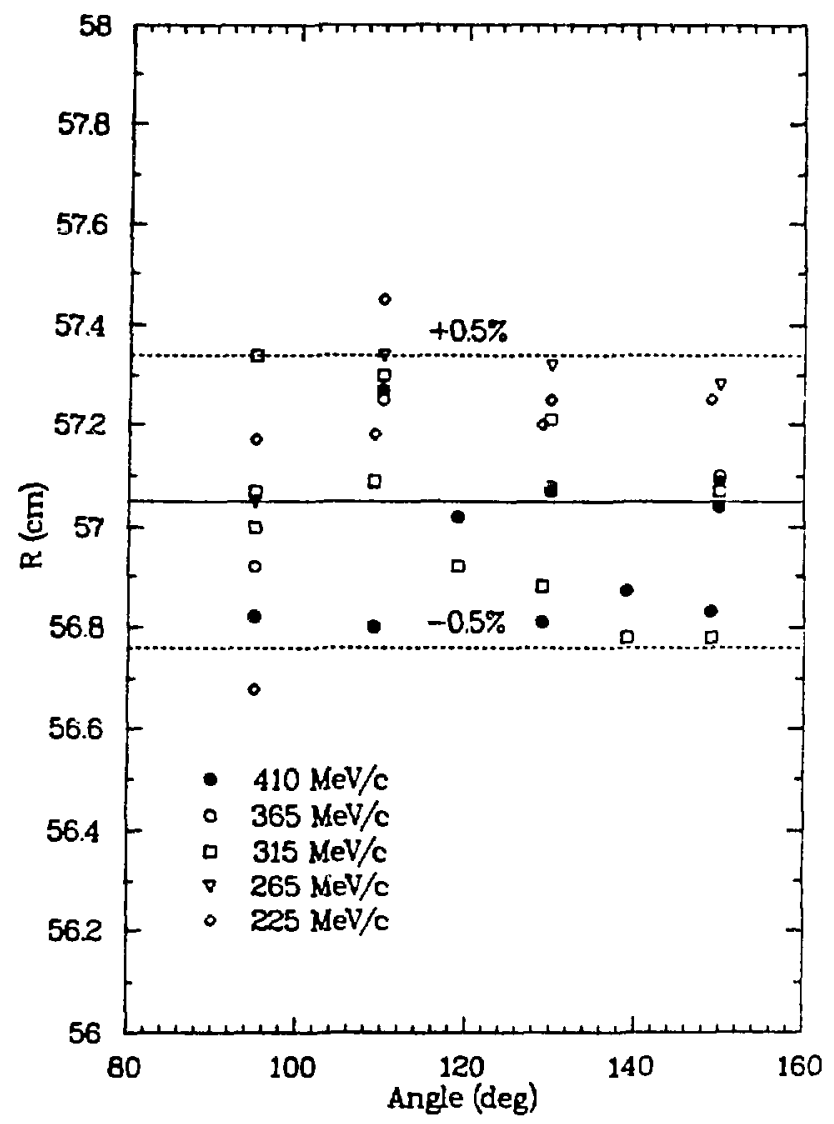

Fig. 7. Measured values of the effective radius $R$ of the circular magnet for the region of the incident beam as a function of scattering angle for an infinitely heavy nucleus. Results are shown for five values of pion momentum. The central value found for $\mathrm{R}=57.05 \mathrm{~cm}$ is indicated by the central horizontal line and the two other lines indicate the values corresponding to $0.5 \%$ above and below this value. 
REFERENCES

1. H. A. Thiessen and S. Sobottka, "A Proposal for EPICS: A High-Resolution Pion Beam and Spectrometer Facility for Nuclear Structure Research at LAMPF," Los Alamos Scientific Laboratory report LA-4534-MS (October 1970).

2. See, for example, K. G. Steffen, High Energy Beam Optics (Interscience, New York, 1965), p. 153. 